\title{
Compostos FENÓlicos TOTAIS E CARACTERÍSTICAS FÍSICO-QUÍMICAS DE FRUTOS DE JABUTICABA
}

\section{Eraldo Santos de Almeida ${ }^{1}$, Rafael José Navas da Silva ${ }^{2}$ e Estela Maria Gonçalves ${ }^{3 *}$}

\author{
${ }^{1}$ Faculdade de Tecnologia de Capão Bonito \\ ${ }^{2}$ Docente do Centro de Ciências Agrágrias, Universidade Federal de Alagoas. \\ ${ }^{3}$ Docente do Centro Estadual de educação Tecnológica Paula Souza, Faculdade de Tecnologia de Capão Bonito. \\ *Autor para correspondência: estela.goncalves@fatec.sp.gov.br
}

Recebido em 29 de agosto de 2016. Aceito em 07 de março de 2018. Publicado em 06 de abril de 2018.

\begin{abstract}
Resumo - A jabuticabeira é nativa da Mata Atlântica e amplamente cultivada em diversas regiões do Brasil e seus frutos apresentam potencial para serem utilizados nas indústrias de alimentos e cosméticos. O objetivo deste trabalho foi avaliar características físico-químicas e compostos fenólicos totais em frutos e frações de frutos de duas espécies de jabuticaba (Myrciaria grandifolia e Myrciaria jabuticaba) da região de Capão Bonito/SP. Foram avaliadas as características físico-químicas de umidade, $\mathrm{pH}$, acidez total titulável e sólidos solúveis e o teor de compostos fenólicos totais em polpa, semente, fruto inteiro e casca. Os resultados mostraram que a umidade, a acidez total titulável e os compostos fenólicos totais da polpa, semente, fruto inteiro e casca foram significativamente maiores nas amostras de M. jabuticaba, enquanto que os teores de sólidos solúveis da polpa e do fruto inteiro foram significativamente maiores em M. grandifolia. Os frutos de jabuticaba das duas espécies estudadas apresentam diferentes características físico-químicas e teores expressivos de compostos fenólicos totais, concentrados especialmente na casca, o que evidencia o seu potencial como fonte de nutrientes e de substâncias antioxidantes.
\end{abstract}

Palavras-chave: Antioxidante; Myrciarla Grandifolda; Myrciarla Jabuticaba.

\section{TOTAL PHENOLIC COMPOUNDS AND PHYSICOCHEMICAL CHARACTERISTICS OF JABUTICABA FRUIT}

Abstract - Jaboticabeira is a native Atlantic Forest species widely cultivated in several regions of Brazil and its fruits have potential to be used in the food and cosmetic industries. The objective of this study was to investigate physicochemical characteristics and total phenolic compounds in fruits and fractions of two species of jabuticaba (Myrciaria grandifolia and Myrciaria jabuticaba) from Capão Bonito/SP. The physicochemical characteristics of moisture, $\mathrm{pH}$, titratable total acidity and soluble solids and total phenolic compounds content in pulp, seed, whole fruit and peel were evaluated. The results showed that moisture, titratable total acidity and total phenolic compounds of pulp, seed, whole fruit and peel were significantly higher in the samples of M. jabuticaba, while the soluble solids contents of the pulp and whole fruit were significantly higher in M. grandifolia. The jabuticaba fruits of the two species have different physicochemical characteristics and expressive contents of total phenolic compounds, especially concentrated in the peel, which highlights its potential as a source of nutrients and antioxidants.

Keywords: ANTIOXIDANT; MYrCLARLA GR ANDIFOLIA; MYRCLARIA JABUTICABA.

\section{COMPUESTOS FENÓLICOS TOTALES Y CARACTERÍSTICAS FÍSICO-QUÍMICAS DE LOS FRUTOS DE JABOTICABA}

Resumen - La jaboticaba es nativa del Bosque Atlántico y ampliamente cultivada en diversas regiones del Brasil y sus frutos tienen potencial para su uso en las industrias de alimentos y cosméticos. El objetivo de este estudio fue evaluar las 
características físico-químicas y compuestos fenólicos totales en frutos y fracciones de frutos de dos especies de jaboticaba (Myrciaria grandifolia y Myrciaria jabuticaba) de la región de Capão Bonito/SP. Se evaluaron las características físico-químicas de humedad, pH, acidez total titulable y sólidos solubles y el contenido de compuestos fenólicos totales en pulpa, semilla, fruto entero y corteza. Los resultados mostraron que la humedad, la acidez titulable y los compuestos fenólicos totales de la pulpa, semilla, fruto entero y la corteza fueron significativamente mayores en muestras de M. jabuticaba, mientras que los contenidos de sólidos solubles de la pulpa y del fruto entero fueron significativamente mayores en M. grandifolia. Los frutos de jaboticaba de las dos especies estudiadas presentan diferentes características físico-químicas y contenidos expresivos de compuestos fenólicos totales, concentrados sobre todo en la corteza, lo que evidencia su potencial como fuente de nutrientes y de sustancias antioxidantes.

Palavras clave: Antioxidante; Myrclarla Grandifolda; Myrciarla Jabuticaba.

\section{INTRODUÇÃO}

A flora brasileira destaca-se pela grande diversidade de árvores frutíferas em todo seu território, exercendo um papel importante para variabilidade que existe dentro do conjunto de espécies vegetais. Entre as plantas de frutos comestíveis podemos citar a jaboticabeira, pertencente à família Myrtaceae, uma frutífera com origem no Centro-Sul do Brasil cultivada do extremo sul ao extremo norte do país (Lorenzi et al. 2006).

A espécie é nativa da Mata Atlântica, apresentando tanto importância socioeconômica, como cultural. Ocorre mais comumente nas regiões Sudeste e Sul e com menor frequência no Centro-Oeste e Nordeste. Apresenta espécies e variedades muito cultivadas, como a Paulista (Myrciaria cauliflora Berg) e a Sabará (Myrciaria jabuticaba (Vell Berg). De grande valor sensorial e nutricional, seus frutos globosos, de polpa suculenta em geral doce, são comumente consumidos in natura ou na forma de doces e geleias (Lorenzi et al. 2006).

Espécies frutíferas da Mata Atlântica estão sendo pesquisadas quanto às propriedades físico-químicas e teores de substâncias antioxidantes, como os compostos fenólicos. Estas substâncias podem atuar na modulação do estresse oxidativo e na proteção sob diversos fitopatógenos (Marin et al. 2008; Bouayed e Bohn 2010, Haminiuk et al. 2011, Hu 2011).

Os polifenóis, que são metabólitos secundários de origem vegetal, apresentam potencial efeito benéfico à saúde devido à sua atividade antioxidante e moduladora do estresse oxidativo (Andrés-Lacueva et al. 2010, $\mathrm{Hu}$ 2011). Esse corresponde a um desequilíbrio entre as reações oxidativas e antioxidativas que ocorrem naturalmente nas células durante o metabolismo aeróbico, favorecendo a oxidação, podendo ocasionar danos a moléculas como DNA, lipídeos, proteínas e carboidratos e estão envolvidos em processos como mutagênese e carcinogênese (Sies 1993).

Neste contexto, substâncias antioxidantes exógenas, como as provenientes da dieta, podem apresentar grande importância na modulação do estresse oxidativo (Barbosa et al. 2010). Assim, pesquisas envolvendo antioxidantes oriundos de fontes naturais têm sido desenvolvidas, devido a sua importância na prevenção do desencadeamento das reações oxidativas tanto nos alimentos como no organismo (Broinizi et al. 2007).

A jabuticaba apresenta frutos de grande valor nutricional, possuindo alto teor de carboidratos, fibras, vitaminas, flavonoides e, ainda, sais minerais como ferro, cálcio e fósforo (Ascheri et al. 2006).

Dessa forma, o presente trabalho tem como objetivo avaliar as características físico-químicas e compostos fenólicos totais em frutos de duas espécies de jabuticaba - Myrciaria grandifolia e Myrciaria jabuticaba, provenientes da região de Capão Bonito/SP. 


\section{Material e MÉtodos}

Frutos das espécies M. grandifolia e M. jabuticaba foram coletados maduros na região de Capão Bonito/ SP, localizada em latitude $24^{\circ} 00^{\prime} 21^{\prime \prime}$ Sul e longitude $48^{\circ} 20^{\prime} 58^{\prime \prime}$ Oeste. Após a coleta, foram acondicionados em sacos plásticos, triados, lavados com água potável e mantidos em freezer até a utilização. No momento do uso, os frutos foram higienizados através de lavagem com água potável, seguida de desinfecção por imersão em solução clorada (hipoclorito de sódio na concentração de $250 \mathrm{mg} \cdot \mathrm{L}^{-1}$ ) durante 15 min com posterior enxágue em água potável, conforme a Portaria CVS-5/2013 (São Paulo 2013). As frações dos frutos (polpa, sementes e cascas) foram obtidas através de separação manual e, a seguir, as frações resultantes e os frutos inteiros foram triturados em liquidificador para a realização das análises em triplicata.

Para análise do teor de umidade, utilizou-se o método de estufa a $105^{\circ} \mathrm{C}$ até atingir o peso constante, com 10 gramas da amostra e os resultados expressos em umidade (\%) ou substâncias voláteis a $105^{\circ} \mathrm{C}$ (Instituto Adolfo Lutz 2008).

Para determinação eletrométrica do pH, os extratos aquosos após homogeneização, foram avaliados e determinado o pH com aparelho (Instituto Adolfo Lutz 2008).

O teor de sólidos solúveis foi determinado nos extratos aquosos por refratometria, utilizando-se um refratômetro manual e os resultados expressos em ${ }^{\circ}$ Brix.

Para determinação da acidez total titulável, aos extratos aquosos foram adicionados $0,3 \mathrm{~mL}$ de solução de fenolftaleína para cada $100 \mathrm{~mL}$ de solução a ser titulada. A titulação foi realizada com solução de hidróxido de sódio 0,1 M sob agitação constante, até coloração rósea persistente por 30 segundos. Os resultados foram expressos em acidez total titulável por cento e em g de ácido cítrico 100. $\mathrm{mL}^{-1}$ (Instituto Adolfo Lutz 2008).

Para avaliação de compostos fenólicos totais foi realizada extração aquosa com pesagem de $5 \mathrm{~g}$ das amostras, diluídas em $20 \mathrm{~mL}$ de água ultrapura em frascos Erlenmeyer e mantidas sob agitação durante $1 \mathrm{~h}$. A seguir, as amostras foram centrifugadas a $3600 \mathrm{rpm}$ durante $30 \mathrm{~min}$. Os sobrenadantes foram coletados e armazenados sob refrigeração, ao abrigo da luz, até o momento das dosagens.

Os compostos fenólicos totais foram determinados com base na metodologia descrita por Keevil et al. (2000). Em um tubo de ensaio foi colocada uma alíquota $(50 \mu \mathrm{L})$ do extrato aquoso da amostra, acrescida de $5 \mathrm{~mL}$ do reagente de Folin-Ciocalteu (diluído em água ultrapura na proporção 1:10, v/v) e $4 \mathrm{~mL}$ de solução de carbonato de sódio $\left(\mathrm{Na}_{2} \mathrm{CO}_{3}\right.$ a 7,5\%, 75 g.L $\left.\mathrm{L}^{-1}\right)$. A amostra foi homogeneizada e mantida ao abrigo da luz por 2 h para leitura da absorbância a $700 \mathrm{~nm}$ em espectrofotômetro. Foi utilizado como padrão o ácido gálico e os resultados expressos em mg equivalentes de ácido gálico $\mathrm{L}^{-1}$. Todas as análises foram realizadas em triplicata e os resultados foram submetidos ao teste t de Student ao nível de $5 \%$ de significância $(\mathrm{p}<0,05)$ para comparação das médias.

\section{RESUltados E Discussão}

Os resultados obtidos para as características físico-químicas dos frutos e frações de frutos das espécies $M$. jabuticaba e M. grandifolia estão expressos na Tabela 1. 
Tabela 1 - Características físico-químicas em frutos e frações de frutos de diferentes espécies de jabuticaba.

\begin{tabular}{lcccccccc}
\hline \multirow{2}{*}{ Análise } & \multicolumn{2}{c}{ Polpa } & \multicolumn{2}{c}{ Semente } & \multicolumn{2}{c}{ Fruto inteiro } & \multicolumn{2}{c}{ Casca } \\
& M. grandifolia & M. jabuticaba & M. grandifolia & M. jabuticaba & M. grandifolia & M. jabuticaba & M. grandifolia & M. jabuticaba \\
\hline Umidade & $87,35 \pm 0,06$ & $89,03 \pm 0,09$ & $56,57 \pm 0,19$ & $60,33 \pm 0,63$ & $82,96 \pm 0,31$ & $85,88 \pm 0,57$ & $81,22 \pm 0,35$ & $82,63 \pm 0,13$ \\
$\mathrm{pH}$ & $3,39 \pm 0,01$ & $3,10 \pm 0,01$ & $3,29 \pm 0,05$ & $4,01 \pm 0,06$ & $3,36 \pm 0,03$ & $3,10 \pm 0,07$ & $3,39 \pm 0,01$ & $3,37 \pm 0,11$ \\
Acidez & $0,31 \pm 0,01$ & $0,73 \pm 0,07$ & $0,45 \pm 0,00$ & $0,72 \pm 0,02$ & $0,45 \pm 0,01$ & $1,19 \pm 0,00$ & $0,42 \pm 0,01$ & $0,79 \pm 0,03$ \\
S. solúveis & $11,33 \pm 0,33$ & $3,17 \pm 0,17$ & $\mathrm{nd}^{*}$ & $1,00 \pm 0,00$ & $11,67 \pm 0,33$ & $2,00 \pm 0,00$ & nd $^{*}$ & $\mathrm{nd}^{*}$ \\
\hline
\end{tabular}

* nd: não detectados

Os teores de umidade (em porcentagem) da polpa, semente, fruto inteiro e casca foram significativamente maiores nas amostras da espécie $M$. jabuticaba (89,03; 60,33; 85,88 e 82,63, respectivamente) em comparação com $M$. gradifolia $(87,35 ; 56,57 ; 82,9$ e 81,22, respectivamente). A jabuticaba apresenta alta perecibilidade após a colheita devido ao alto teor de umidade e de açúcares da polpa, ocasionando restrito período de comercialização in natura, estimado em torno de dois dias, incentivando estudos sobre técnicas de conservação e métodos de processamento dos frutos (Asquieri et al. 2004; Brunini et al. 2004; Citadin et al. 2005; Ascheri et al. 2006). De acordo com Chitarra e Chitarra (2005) a água é o componente mais abundante nos produtos hortícolas, perfazendo até $95 \%$ dos mesmos, de acordo com o produto, com o suprimento dado aos tecidos à época da colheita, bem como, com a temperatura e a umidade relativa do meio ambiente. Nas frutas pode apresentarse em uma faixa entre 65\% a 95\% (Cecchi 2003). As frutas e hortaliças perdem o suprimento de água quando colhidas, murchando rapidamente se mantidas em ambiente com umidade relativa baixa e temperatura elevada. Além disso, de acordo com Araújo et al. (2010) os teores de umidade de $M$. jabuticaba diminuem ao longo do desenvolvimento, variando de 81,2 a $76,9 \%$ na casca e 85,0 a 79,7\% na polpa, do $25^{\circ}$ ao $50^{\circ}$ dia de desenvolvimento dos frutos.

A determinação da umidade é uma das medidas mais importantes e utilizadas na análise de alimentos, estando relacionada com sua estabilidade, qualidade e composição, podendo afetar a estocagem, a embalagem e o processamento.

Os teores de umidade em frutos de jabuticaba e em suas frações geralmente apresentam-se elevados, tais como os obtidos neste trabalho. Abe et al. (2012) observaram umidade de aproximadamente $86 \%$ na casca e 85\% na polpa+semente de frutos de M. jabuticaba. Em M. cauliflora, a umidade da polpa obtida após peneiração de frutos maduros foi de $87,67 \%$ (Nunes et al. 2014).

Em outro estudo, teores de umidade (em g. $100 \mathrm{~g}^{-1}$ ) do fruto inteiro, casca, polpa e semente, respectivamente, foram de 80,35; 75,84; 83,91 e 70,40 para frutos da variedade Paulista. Na variedade Sabará, os teores foram iguais a 79,41; 84,24; 84,95 e 71,48, observando-se alto conteúdo de água, tanto no fruto inteiro quanto nas frações de ambas as variedades de jabuticaba (Lima et al. 2008), corroborando os dados obtidos neste estudo para as espécies M. jabuticaba e M. grandifolia.

Os valores de $\mathrm{pH}$ foram significativamente maiores nas amostras de polpa e fruto inteiro da espécie $M$. grandifolia e nas amostras de semente de $M$. jabuticaba. Os resultados médios de $\mathrm{pH}$ das amostras de casca não foram diferentes entre as duas espécies. A medida de $\mathrm{pH}$ refere-se ao potencial hidrogeniônico da amostra e, de acordo com Cecchi (2003), é importante para determinação da deterioração do alimento com crescimento de microrganismos, atividade de enzimas, textura de geleias e gelatinas, retenção do sabor-odor de produtos e 
verificação do estado de maturação de frutas e implica na escolha do tipo de embalagem. Como foi demonstrado pelos resultados obtidos, podem ser considerados quimicamente como ácidos os frutos e as frações dos frutos das duas espécies de jabuticaba avaliadas. Para fins industriais, poderia representar um bom fator, retardando possíveis alterações microbianas e prolongando a vida útil de produtos subsequentes (Nunes et al. 2014).

Valores de $\mathrm{pH}$ ácidos em jabuticaba também foram obtidos por outros autores. $\mathrm{Na}$ espécie $M$. cauliflora foi determinado pH igual a 3,14 em amostras de polpa (Nunes et al. 2014). Comparando duas variedades de jabuticaba, os valores de $\mathrm{pH}$ apresentaram pequena variação entre as frações casca, fruto inteiro, polpa e semente entre as variedades Paulista e Sabará (Lima et al. 2008). As variações observadas nos teores de pH entre as amostras da espécie $M$. jabuticaba e $M$. grandifolia podem estar relacionadas a condições genéticas, ambientais, como também a outros fatores. A região de cultivo interfere nas características físico-químicas de frutos de jabuticaba. Oliveira et al. (2003) caracterizaram frutos de M. jabuticaba provenientes de diferentes regiões do Estado de São Paulo e observaram que, em sua polpa, o pH variou de 2,91 a 3,70, dependendo da origem dos frutos.

Além do processamento e da região de cultivo, o estádio de desenvolvimento dos frutos também se reflete em alterações nas características físico-químicas. Araújo et al. (2010) observaram elevação do pH, de 2,7 até 3,6, no período do $25^{\circ}$ ao $50^{\circ}$ dia de desenvolvimento de frutos de M. jabuticaba.

A acidez total titulável da polpa, semente, fruto inteiro e casca foram significativamente maiores nas amostras da espécie $M$. jabuticaba $(0,73 ; 0,72 ; 1,19$ e 0,79 , respectivamente) em comparação com $M$. gradifolia $(0,31 ; 0,45 ; 0,45$ e 0,42 , respectivamente). Os ácidos orgânicos presentes em alimentos influenciam o sabor, o odor, a cor, a estabilidade e a manutenção da qualidade. A acidez titulável de frutas varia de 0,2 a $0,3 \%$ em frutas de baixa acidez até $6 \%$ em frutas ácidas. Produtos mais ácidos são naturalmente mais estáveis quanto à deterioração e a proporção relativa de ácidos orgânicos presente em frutas e vegetais varia com o grau de maturação e com as condições de crescimento (Cecchi 2003), e com o amadurecimento, geralmente, os frutos perdem rapidamente a acidez (Chitarra e Chitarra 2005).

Corroborando os dados obtidos neste estudo para as amostras da espécie M. jabuticaba, Oliveira et al. (2003) caracterizaram M. jabuticaba de diferentes regiões de cultivos do estado de São Paulo e observaram que, em sua polpa, a acidez total titulável variou de 0,888 a 1,625. De forma semelhante, o valor encontrado de acidez foi de 1,09\% de ácido cítrico 100.g-1 de polpa de M. cauliflora (Nunes et al. 2014).

Lima et al. (2008) em comparação de duas variedades de jabuticaba, Paulista e Sabará, observaram que a acidez total titulável variou entre as frações casca, fruto inteiro, semente e polpa, sendo igual apenas na casca e semente da variedade Sabará. As sementes apresentaram os maiores teores de acidez, sendo maior na variedade Paulista, e as polpas obtiveram os menores valores. De maneira similar à avaliação do pH, as variações observadas na acidez das amostras das espécies $M$. jabuticaba e $M$. grandifolia podem estar relacionadas a diversidade genética (Zerbielli et al 2016), grau de maturação e condições de armazenamento dos frutos (Vieites et al. 2011), características climáticas (Guedes et al. 2014), manejo das plantas (Wagner Júnior et al. 2017) e local de cultivo (Oliveira et al. 2003).

Para os teores de sólidos solúveis, os valores obtidos na polpa e no fruto inteiro foram significativamente maiores nas amostras da espécie M. grandifolia. Os teores de sólidos solúveis dependem da espécie em estudo, como também de outros fatores, como cultivar, maturação e clima. Os valores médios comumente estão entre 8\% e 14\%; porém, podem apresentar uma ampla variação, entre 2\% e 25\% (Chitarra e Chitarra 2005). Em 
polpa da espécie $M$. caulifora foi observada concentração de sólidos solúveis de 14,2 ${ }^{\circ}$ Brix (Nunes et al. 2014). Em outro estudo, Oliveira et al. (2003) ao caracterizarem M. jabuticaba provenientes de diferentes regiões de cultivo observaram que, em sua polpa, o teor de sólidos solúveis totais variou de 11,5 a 17,9 ${ }^{\circ}$ Brix. Avaliando duas variedades de jabuticaba, Lima et al. (2008) identificaram que a polpa das variedades Paulista e Sabará apresentaram os maiores teores de sólidos solúveis totais, em comparação com semente, casca e fruto inteiro.

Os sólidos solúveis na polpa de M.jabuticaba variam de acordo com os diferentes estádios de desenvolvimento, variando de $7,6^{\circ}$ Brix a $16,8^{\circ}$ Brix no período entre o $25^{\circ}$ ao $50^{\circ}$ dias de desenvolvimento dos frutos. A elevação do teor de sólidos solúveis estabiliza-se aos $16^{\circ}$ Brix no final do período de maturação do fruto (43 a 50 dias); este comportamento possivelmente está associado a reações químicas resultantes da respiração, que tem como principais substratos os carboidratos e ácidos orgânicos. Assim, a partir do $43^{\circ}$ dia após o florescimento, os frutos de $M$. jabuticaba apresentam características físicas e químicas ideais de qualidade apresentando uma redução da acidez, elevação no pH e do teor de sólidos solúveis, tornando o fruto neste estádio de desenvolvimento com melhor potencial para o consumo in natura como para conservação e industrialização (Araújo et al. 2010).

Com relação aos teores de compostos fenólicos totais, esses podem ser observados na Tabela 2 para ambas as espécies. Os teores de compostos fenólicos totais na polpa, semente, fruto inteiro e casca foram significativamente maiores nas amostras da espécie M. jabuticaba. Nos frutos, os compostos fenólicos participam no "flavor", na coloração, na vida de prateleira e na sua ação como alimentos funcionais, especialmente como antioxidantes, apresentando correlação positiva com a capacidade antioxidante dos mesmos e são normalmente encontrados em folhas, sementes e frutos, em concentrações variadas dependendo da espécie, cultivar e parte do fruto (Chitarra e Chitarra 2005). Em geral, esses compostos estão associados ao mecanismo de adaptação e resistência das plantas ao meio ambiente, podendo influenciar no sabor, nas características tecnológicas, assim como no potencial nutricional e funcional de espécies frutíferas (Rocha et al. 2011).

Tabela 2 - Compostos fenólicos totais em frutos e frações de frutos de diferentes espécies de jabuticaba.

\begin{tabular}{lcc}
\hline Amostra & Myrciaria jabuticaba & Myrciaria grandifolia \\
\hline Polpa $^{1}$ & $1480,57 \pm 46,46$ & $249,82 \pm 26,14$ \\
Semente $^{1}$ & $5505,27 \pm 461,50$ & $2960,66 \pm 126,70$ \\
Fruto inteiro $^{1}$ & $4053,23 \pm 220,30$ & $2305,04 \pm 93,30$ \\
Casca $^{1}$ & $6070,02 \pm 395,60$ & $4512,07 \pm 87,14$ \\
\hline
\end{tabular}

${ }^{1}$ Resultados expressos em mg equivalentes de ácido gálico $\mathrm{L}^{-1}$

De acordo com Lima et al. (2008) a casca da variedade Sabará apresenta maiores teores de compostos fenólicos totais quando comparada a variedade Paulista. Abe et al. (2012) observaram teor de compostos fenólicos totais de 7,44 g. $\mathrm{kg}^{-1} \mathrm{em}$ fruto inteiro de $M$. jabuticaba. Em outra análise, os autores avaliaram frutos de M. jabuticaba coletados em 5 graus de maturação, de acordo com a cor da casca: verde, verde com pontos vermelhos, vermelha, roxa ou roxa escura, que corresponderia ao ponto máximo de maturação. O teor de compostos fenólicos totais diminuiu conforme a maturação dos frutos, com maiores concentrações no fruto com a casca verde até concentrações menores no fruto com a casca roxa escura, demonstrando que a maturação é fator determinante na concentração de polifenóis presentes nos frutos da espécie M. jabuticaba. Além disso, independente do grau de maturação, os autores demonstraram que os polifenóis estão concentrados na casca dos frutos, quando comparada com a polpa e a semente, corroborando assim os resultados obtidos neste estudo. 
As diferenças observadas entre as espécies $M$. jabuticaba e M. grandifolia podem estar relacionadas a diversos fatores. Diferentes condições podem afetar as concentrações de compostos fenólicos, como, por exemplo, fatores genéticos, condições ambientais e grau de maturidade dos frutos, bem como o processamento e o armazenamento (Sánchez-Moreno 2002). Os fenólicos variam também com a espécie, a cultivar, o grau de maturação, local de cultivo e a estação do ano (Chitarra e Chitarra 2005), podendo ser esse o fator das diferenças observadas.

\section{CoNSIDERAÇõES FINAIS}

Os frutos de M. jabuticaba e M. grandifolia provenientes da região de Capão Bonito/SP apresentam diferenças em suas características físico-químicas quanto às variáveis umidade, $\mathrm{pH}$, acidez total titulável e sólidos solúveis.

Apresentam também teores consideráveis de compostos fenólicos totais, tanto nas frações dos frutos como no fruto inteiro, concentrados especialmente nas cascas, indicando o seu potencial como fontes de antioxidantes naturais.

O estímulo de sua produção e consumo pode proporcionar, além de uma fonte de renda, nutrientes e compostos fenólicos importantes na alimentação.

\section{REFERÊNCIAS}

Abe LT, Lajolo, FM, Genovese MI. 2012. Potential dietary sources of ellagic acid and other antioxidants among fruits consumed in Brazil: Jabuticaba (Myrciaria jabuticaba (Vell. berg). Journal of the Science of Food and Agriculture, 92(8):1679-1687.

Ascheri DPR, Ascheri JLR, Carvalho, CWP. 2006. Caracterização da farinha de bagaço de jabuticaba e propriedades funcionais dos extrusados. Ciência e Tecnologia de Alimentos, 26(4):897-905.

Asquieri ER, Candido MA, Damiani C, Assis EM. 2004. Fabricación de vino blanco y tinto de jabuticaba (Myrciaria jaboticaba Berg) utilizando la pulpa y la cáscara respectivamente. Alimentaria, 355:97-109.

Andrés-Lacueva C, Medina-Remon A, Llorach R, Urpi-Sarda M, Khan N, Chiva-Blanch G, Zamora-Ros R, Rotches-Ribalta M, Lamuela-Raventós RM. 2010. Phenolic Compounds: chemistry and occurrence in fruits and vegetables. In: Rosa LA et al. (Eds.), Fruit and vegetable phytochemicals: chemistry, nutritional value and stability, Singapore: Wiley-Blackwell, p. 53-88.

Araújo FMMC, Machado AV, Lima HC, Chitarra AB. 2010. Alterações físicas e químicas do fruto da jaboticabeira (Myrciaria jabuticaba Berg cv. Sabará) durante seu desenvolvimento. Revista Verde, 5(2):109-116.

Barbosa KBF, Costa NMB, Alfenas RCG, Paula SO, Minim VPR, Bressan J. 2010. Estresse oxidativo: conceito, implicações e fatores modulatórios. Revista de Nutrição, 23(4):629-643.

Bouayed J, Bohn T. 2010. Exogenous antioxidants - Double-edged swords in cellular redox state: health beneficial effects at physiologic doses versus deleterious effects at high doses. Oxidative Medicine and 


\section{Cellular Longevity, 3(4):228-237.}

Broinizi PRB, Andrade-Wartha ERS, Silva AMO, Novoa AJV, Torres RP, Azeredo HMC, Alves RE, Mancini-Filho J. 2007. Avaliação da atividade antioxidante dos compostos fenólicos naturalmente presentes em subprodutos do pseudofruto de caju (Anacardium occidentale L.). Ciência e Tecnologia de Alimentos, 27(4):902-908.

Brunini MA, Oliveira AL, Salandini CAR, Bazzo FR. 2004. Influência de embalagens e temperatura no armazenamento de jabuticabas (Myrciaria jabuticaba (Vell) Berg) cv 'SABARÁ'. Ciência e Tecnologia de Alimentos, 24(3):378-383.

Cecchi HM. 2003. Fundamentos teóricos e práticos em análise de alimentos. Campinas: Editora da Unicamp, 208 p.

Chitarra MIF, Chitarra AB. 2005. Pós-colheita de frutas e hortaliças: fisiologia e manuseio. Lavras: Ed. UFLA, 784 p.

Citadin I, Vicari IJ, Silva TT, Danner MA. 2005. Qualidade de frutos de jabuticabeira (Myrciaria cauliflora) sob influência de duas condições de cultivo: sombreamento natural e a pleno sol. Revista Brasileira de Agrociência, 11(3):373-375.

Guedes MNS, Rufini JCM, Azevedo AM, Pinto NAVD. 2014. Fruit quality of jabuticaba progenies cultivated in a tropical climate of altitude. Fruits, 69(6):449-458.

Haminiuk CWI, Plata-Oviedo MSV, Guedes AR, Stafussa AP, Bona E, Carpes ST. 2011. Chemical, antioxidant and antibacterial study of Brazilian fruits. International Journal of Food Science \& Technology, 46(7):15291537.

Hu ML. 2011. Dietary polyphenols as antioxidants and anticancer agents: more questions than answers. Chang Gung Medical Journal, 34:449-460.

Instituto Adolfo Lutz. 2008. Métodos físico-químicos para análise de alimentos, São Paulo: Instituto Adolfo Lutz, 1020 p.

Keevil JG, Osman HE, Reed JD, Folts JD. 2000. Grape juice, but not orange juice or grapefruit juice, inhibits human platelet aggregation. Journal of Nutrition, 130:53-56.

Lima AJB, Corrêa AD, Alves APC, Abreu CMP, Dantas-Barros AM. 2008. Caracterização química do fruto jabuticaba (Myrciaria cauliflora Berg) e de suas frações. Archivos Latinoamericanos de Nutrición, 58(4):416421.

Lorenzi H, Bacher L, Lacerda M, Sartori S. 2006. Frutas Brasileiras e Exóticas Cultivadas de consumo in natura, Nova Odessa: Instituto Plantarum de Estudos da Flora, 640 p.

Marin R, Apel MA, Limberger RP, Raseira MCB, Pereira JFM, Zuanazzi JAS, Henriques AT. 2008. Volatile components and antioxidant activity from some Myrtaceous fruits cultivated in Southern Brasil. Latin American Journal of Pharmacy, 27(2):172-177. 
Nunes JS, Castro DS, Sousa FC, Silva LMM, Gouveia JPG. 2014. Obtenção e caracterização físico-química de polpa de jabuticaba (Myrciaria cauliflora Berg) congelada. Revista Verde, 9(1):234-237.

Oliveira AL, Brunini MA, Salandini CAR, Bazzo FR. 2003. Caracterização tecnológica de jabuticabas 'Sabará' provenientes de diferentes regiões de cultivo. Revista Brasileira de Fruticultura, 25(3):397-400.

Rocha WS, Lopes RM, Silva DB, Vieira RF, Silva JP, Agostini-Costa TS. 2011. Compostos fenólicos totais e taninos condensados em frutas nativas do cerrado. Revista Brasileira de Fruticultura, 33(4):1215-1221.

Sánchez-Moreno C. 2002. Compuestos polifenólicos: estructura y clasificación, presencia en alimentos y consumo, biodisponibilidad y metabolismo. Alimentaria, 2:19-28.

São Paulo. 2013. Portaria CVS-5/2013 de 19 abr de 2003. Regulamento técnico sobre boas práticas para estabelecimentos comerciais de alimentos e para serviços de alimentação, e o roteiro de inspeção. Diário Oficial do Estado, São Paulo, 19 ab 2013.

Sies H. 1993. Strategies of antioxidant defense. European Journal of Biochemistry, 215(2):213-19.

Vieites RL, Daiuto ER, Moraes MR, Neves LC, Carvalho LR. 2011. Caracterização físico-química, bioquímica e funcional da jabuticaba armazenada sob diferentes temperaturas. Revista Brasileira de Fruticultura, 33(2):362375.

Wagner Júnior A, Paladini MV, Danner MA, Moura GC, Guollo K, Nunes IB. 2017. Aspects of the sensorial quality and nutraceuticals of Plinia cauliflora fruits. Acta Scientiarum, 39(4):475-485.

Zerbielli L, Nienow AA, Dalacorte L, Jacobs R, Daronch T. 2016. Diversidade físico-química dos frutos de jabuticabeiras em um sítio de ocorrência natural. Revista Brasileira de Fruticultura, 38(1):107-116. 